\title{
The Validity of Behavior Against Drought Disaster of Farm Households in Yunnan Mountain = Based on Different Body Discriminant Angles
}

\author{
Min Tian ${ }^{1,2}$, Yimeng Cheng ${ }^{1}$, Jianlan $\mathrm{Li}^{1}$, Shiliang $\mathrm{Hu}^{1}$, Xinhui $\mathrm{Xu}^{1}$ \\ ${ }^{1}$ Yunnan University College of Resource Environment and Earth Science, Kunming 650091, China \\ ${ }^{2}$ Yunnan Institute of Geography, Yunnan University, Kunming 650091, China

\section{云南山地农户旱灾应灾行为有效性研究 二一基于不同主体判识角度} \\ 田敏 ${ }^{1,2}$, 程怡萌 ${ }^{1}$, 李建兰 ${ }^{1}$, 胡世亮 ${ }^{1}$, 许新慧 ${ }^{1}$ \\ 1云南大学资源环境与地球科学学院, 云南昆明, 650091, 中国 \\ 2 云南省地理研究所, 云南昆明, 650091, 中国
}

\begin{abstract}
To analysis the efficient of farmer's disaster preparedness behaviors, we built and compared efficient index of two models. The one model's subject was experts and calculated by AHP (Analytic Hierarchy Process), and another subject was farmers and processed by TOPSIS (Technique for Order Preference by Similarity to an Ideal Solution). The results showed that the experts and farmers have broadly equal assessment on the importance of 12 different disaster preparedness behaviors. At the same time, the two models have a difference in some behaviors such as buying water, engaging in a variety of income-generating activities and purchasing water receivers. By identifying and comparing from the two models, the amounts of different types of disaster preparedness farmers were almost same. Moreover, the second types of two models were similar while the first and the third types were slightly different. And some farmers belong to the same type may undertake
\end{abstract}

第一作者电邮: tianmin@ynu.edu.cn different disaster preparedness behaviors.

Keywords: Drought disaster; Disaster response body; Disaster preparedness behavior; Validity

摘要

本文主要从专家和农户两个不同研究主体出 发, 分别运用层次分析法和 TOPSIS 分析法进 行对比研究, 并且构建应灾行为有效性指数, 对农户应灾行为有效性进行了分析研究。通过 比对分析, 结果表明: 专家和农户对 12 种不 同应灾行为重要性的评价大致相同; 但是对买 水、从事多种收入活动和购买储水物品等应灾 行为还存在一定的差异。同时, 从专家的角度 和从农户的角度判识, 根据应灾行为有效性指 数划分的应灾类型的数量大致相同; 特别是对 应灾类型 II 农户的划分接近一致, 应灾行为 I 和应灾行为III划分的人数还有一定差异。同 时, 属于同一种应灾行为类型不同农户采取的 应灾行为存在差异。

关键词: 旱灾; 应灾主体; 应灾行为; 有效性

1. 引言

目前, 国内关于对农户应灾行为的研究主 要有: 孙雪萍等学者 ${ }^{[1]}$ 通过构建云南省 2009 
Risk Analysis and Crisis Response in Big Data Era (RAC-16)

-2010 年气象干旱等级的演变序列, 以及不 同社会主体(政府、社会组织团体、灾民) 的应 灾过程序列, 以此分析云南旱灾的应灾情况。 谢家智和周振 ${ }^{[2]}$ 分析了由于受非贝叶斯法则、 代表性法则、针定效应、从众行为和框架效应 等的外界偏差性行为显著干扰的情况下, 农民 非理性选择很大程度上会主导个体的行为, 农 民极易出现风险偏好的倾向。刘文方等学者 ${ }^{[3]}$ 基于系统的观点分析灾害链, 并针对灾害链系 统对一些断链减灾模式进行讨论。孙莉莉等学 者 ${ }^{[4]}$ 通过 KMO 指数、球形检验和主成份分析方 法, 分别对台风灾害的行为模式和影响因素进 行了信效度分析。陈利和谢家智 ${ }^{[5]}$ 运用 Logistic 模型来分析农户对农业灾害赔偿满 意度的影响因素以及农户对农业灾害的认识 和行为。通过研究发现, 关于农业灾害的研究 主体大部分以农户为主, 并且研究方法以定性 描述为主很少有涉及到具体方法的定量研究。 本文分别从专家和农户不同识别主体分析旱 灾应灾行为的有效性, 并且分别运用层次分析 法和 TOPSIS 分析法 ${ }^{[6-8]}$ 对其进行对比研究。

\section{2. 数据来源与研究思路}

\section{1. 数据来源}

本次田野调查的对象是云南山地农户, 笔 者于 2015 年1月到南涧县进行相关的问卷调查 和现场访谈。本次研究共发放 235 份问卷, 其 中的 20 份为预调查, 回收问卷 204 份, 剔除无 效问卷, 最后得到有效问卷 190 份, 有效率为 $80.9 \%$ 。其中, 农户问卷 175 份, 分别为红星村 59 份, 拥翠村 71 份, 杏子山村 45 份 (该村总人
口较少); 专家打分15份。通过此次问卷也得 到了农户应对旱灾常用的 12 种应灾行为方法 （见表1）。

\section{2. 重要程度评价准则}

为方便数据信息的搜集和统计分析, 农户 应灾行为测评指标主要采用态度量化方法, 问 卷采用李克特量表法赋值, 每个应灾行为分别 根据农户对其重要程度的评价而赋分。关于上 述测度农户的 12 种应灾行为, 在此先说明各 类应灾行为重要程度评价的准则。应灾行为指 标重要程度判定的标准主要有两个:

一是农户采取的应灾行为有利于农户家 庭收入的稳定或在旱灾年份收入基本保持不 变;

二是农户采取的应灾行为有利于农产品 生产安全和农业的可持续发展。农户采取的应 灾行为越有利于以上几个方面, 则其重要程度 越高。

\section{3. 研究思路}

本文主要从专家和农户两个不同主体对 农户应灾行为有效性进行了分析研究。从专家 角度分析, 主要采用层次分析法得到各个应灾 行为的指标权重值; 而由于农户的与专家文化 程度与理解能力的差别本文采用 TOPSIS 分析 法进行研究, 得到各个应灾行为的综合评价 值; 然后将两个方法得到的结果对比分析。最 后, 分别利用指标权重值和综合评价值建立应 灾行为有效性指数, 然后从专家层面和农户层 面分析对比二者的差异及原因。技术路线见图 1.

表1. 关于旱灾主要的应灾行为

\begin{tabular}{c|c|c|c}
\hline 序号 & 应灾行为 & 序号 & 应灾行为 \\
\hline $\mathrm{X} 1$ & 打井修水㟶 & $\mathrm{X} 7$ & 地膜覆盖 \\
\hline $\mathrm{X} 2$ & 浇灌设施准备 & $\mathrm{X} 8$ & 育苗等田间管理行为 \\
\hline $\mathrm{X} 3$ & 购买储水物品 & $\mathrm{X} 9$ & 购买农作物保险 \\
\hline $\mathrm{X} 4$ & 买水 & $\mathrm{X} 10$ & 从事多种收入活动 \\
\hline $\mathrm{X} 5$ & 多样化种植 & $\mathrm{X} 11$ & 弃耕并外出务工 \\
\hline $\mathrm{X} 6$ & 种植耐早或节水农作物 & $\mathrm{X} 12$ & 祷告祭祀神灵 \\
\hline
\end{tabular}


Risk Analysis and Crisis Response in Big Data Era (RAC-16)

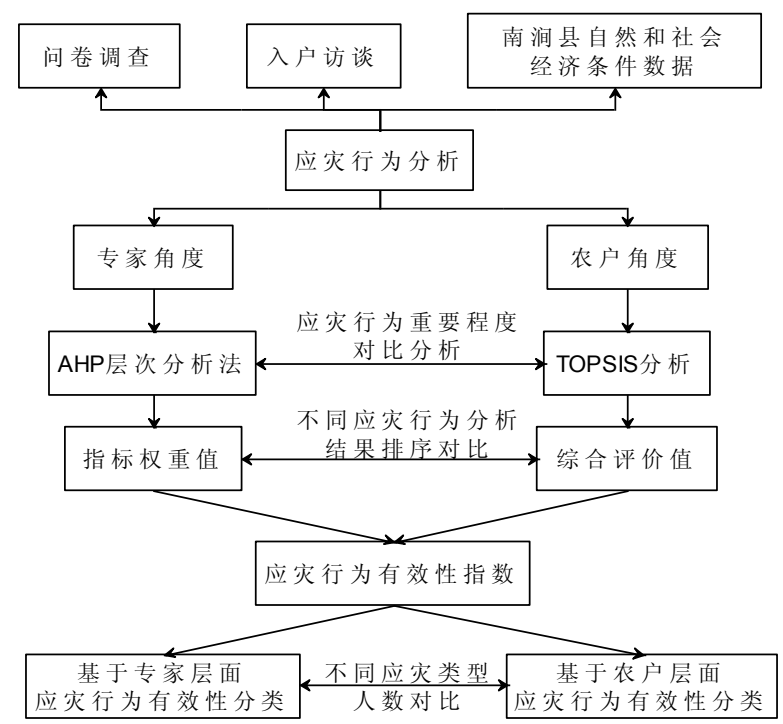

图 1. 技术路线

\section{3. 结果与分析}

\section{1. 应灾行为重要性对比研究}

\subsection{1. 从专家角度分析}

选取15名专家对旱灾来临时所采取的应 灾行为进行评价, 运用层次分析法 ${ }^{[9-10]}$ 对其评 价结果进行分析。具体的计算过程如下:

第一，建立农户应灾行为响应指标体系。 通过查阅文献资料及实地考察，我们发现农户

层面的应灾行为主要表现为耕作生产条件改 良行为、耕作行为和其它行为。农户的耕作生 产条件改良行为主要有：打井、修水空 (塘); 浇灌设施准备; 购买储水物品; 买水。耕作行 为主要有: 多样化种植; 种植耐旱或节水农作 物; 地膜覆盖; 育苗、抢栽抢种、补 (移) 栽 补种、抢收等田间管理行为。其它行为有: 购 买农作物保险; 从事多种收入活动; 弃耕并外 出务工; 祷告、祭祀神灵。因此, 将农户应灾 行为响应涉及的因素进行分层, 通过各指标的 相互关系，建立的指标体系如图2所示。

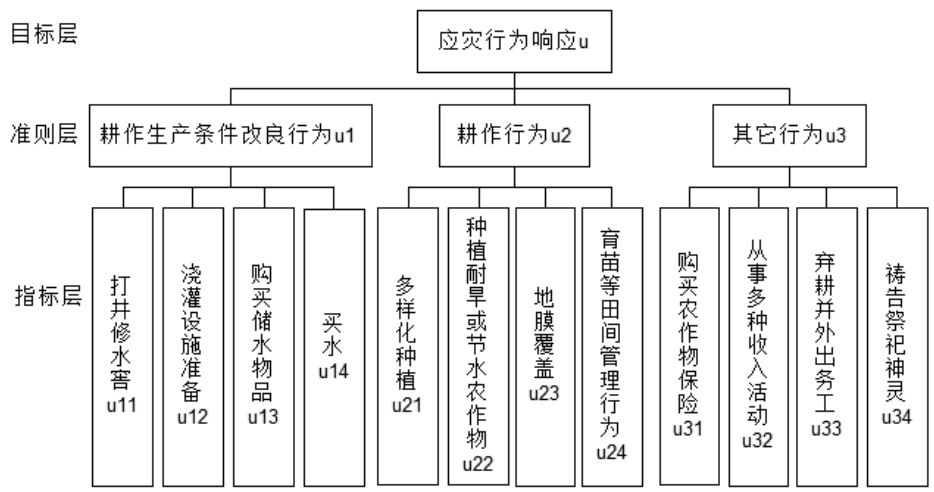

图2. 应灾行为响应指标体系 
Risk Analysis and Crisis Response in Big Data Era (RAC-16)

第二, 构建判断矩阵。在指标体系建立的 基础上, 本研究采用德尔菲法对 15 名专家进 行问卷调查, 通过调查得出专家对各指标重要 性赋值均值, 通过专家组赋值的均值, 得出专 家组对指标的重要性综合评价。同时结合 1 9 标度法 ${ }^{[11]}$ 可得以下判断矩阵:

$$
\begin{aligned}
A & =\left[\begin{array}{ccc}
1 & 1 & 2 \\
1 & 1 & 2 \\
1 / 2 & 1 / 2 & 1
\end{array}\right] \\
A_{1} & =\left[\begin{array}{cccc}
1 & 2 & 3 & 1 \\
1 / 2 & 1 & 2 & 1 / 2 \\
1 / 3 & 1 / 2 & 1 & 1 / 3 \\
1 & 2 & 3 & 1
\end{array}\right] \\
A_{2} & =\left[\begin{array}{cccc}
1 & 1 / 3 & 1 & 1 / 2 \\
3 & 1 & 3 & 2 \\
1 & 1 / 3 & 1 & 1 / 2 \\
2 & 1 / 2 & 2 & 1
\end{array}\right] \\
A_{3} & =\left[\begin{array}{cccc}
1 & 1 / 2 & 1 & 2 \\
2 & 1 & 2 & 3 \\
1 & 1 / 2 & 1 & 2 \\
1 / 2 & 1 / 3 & 1 / 2 & 1
\end{array}\right]
\end{aligned}
$$

第三, 计算各指标权重。首先, 计算判断 矩阵每行元素的乘积 $M_{i}$; 其次, 计算 $M_{i}$ 的n次方 根 $\bar{W}_{i}$; 最后, 将向量 $\left[\begin{array}{llll}\bar{W}_{1} & \bar{W}_{2} & \ldots & \bar{W}_{n}\end{array}\right]^{\mathrm{T}}$ 归一化, 计算各指标的权重 $W_{i}$ 。同时, 运用公式 $\lambda=\frac{1}{n} \sum_{i=1}^{n} \frac{(A \cdot \omega)_{i}}{\omega_{i}}$ 计算最大特征根 $\lambda$; 运用公 式 $C I=\frac{\lambda-n}{n-1}$ 计算一致性指标 $C I$ 。按此方法求 得的指标层其它指标权重, 计算结果如表2所 示。

通过以上分析可以得出, 专家综合认为种 植耐旱或节水农作物、打井修水空和买水是减 轻旱灾的重要应灾行为; 同时, 他们认为购买 农作物保险、弃耕并外出打工、购买储水物品 和祷告祭祀神灵不能够很好的缓解旱情。

\subsection{2. 从农户角度分析}

选取175名农户对旱灾来临时所采取的应 灾行为进行评价, 考虑到农户与专家的文化程

\begin{tabular}{|c|c|c|c|c|c|c|c|}
\hline 准则层 & 权重 & $\lambda_{\max }$ & $\mathrm{CR}$ & 指标层 & 单层权重 & 综合权重 & 排序 \\
\hline 耕作生 & & & & 打井修水窝 & 0.3778 & 0.1511 & 2 \\
\hline 产条件 & 0.4 & 4.1186 & 0.0439 & 浇灌设施准备 & 0.1651 & 0.0660 & 5 \\
\hline 改良行 & & & & 购买储水物品 & 0.0793 & 0.0317 & 8 \\
\hline \multirow[t]{2}{*}{ 为 } & & & & 买水 & 0.3778 & 0.1511 & 2 \\
\hline & & & & 多样化种植 & 0.1105 & 0.0442 & 6 \\
\hline 耕作 & 0.4 & 4.1125 & 0.0417 & 种植耐旱或节水农作物 & 0.5261 & 0.2104 & 1 \\
\hline \multirow[t]{3}{*}{ 行为 } & & & & 地膜覆盖 & 0.1105 & 0.0442 & 6 \\
\hline & & & & 育苗等田间管理行为 & 0.2529 & 0.1012 & 3 \\
\hline & & & & 购买农作物保险 & 0.2116 & 0.0423 & 7 \\
\hline \multirow{3}{*}{$\begin{array}{l}\text { 其它 } \\
\text { 行为 }\end{array}$} & 0.2 & 0.0984 & 0.0364 & 从事多种收入活动 & 0.4844 & 0.0969 & 4 \\
\hline & & & & 弃耕并外出打工 & 0.2116 & 0.0423 & 7 \\
\hline & & & & 祷告祭祀神灵 & 0.0924 & 0.0185 & 9 \\
\hline
\end{tabular}
度、理解能力的差异, 本文运用TOPSIS 分析 法 ${ }^{[12-14]}$ 对其评价结果进行分析。具体的计算过

表 2. 指标层的层次总排序

$\lambda_{\max }=3, \quad C I=0, \quad R I=0.58, \quad C R=0<0.1$ 
程如下:

首先, 运用公式 ${ }^{a_{i j}}=\frac{x_{i j}}{\sqrt{\sum_{i=1}^{n} x_{i j}^{2}}}$, 对各项指标进

行归一化处理。其中 $x_{i j}$ 表示面对 12 种应灾行

为 175 名农户对其重要程度的评价值, 由此可 以得到 $12 \times 175$ 的归一化矩阵; 其次, 进行加权 处理, 即依据各项指标的重要程度分配权值 $W_{i j}$, 在此采用统一的权值, 即令 $W_{i j}$ 为 $12 \times 175$ 的单位矩阵, 通过公式 $Z_{i j}=W_{i j} \times A_{i j}$ 运算得到各 项指标加权矩阵值; 再通过从矩阵Z中选出各 项指标的参数值的最大值和最小值, 确定最优 方案和最差方案。然后, 分别按公式 $D_{i}^{ \pm}=\sqrt{\sum_{j=1}^{n}\left(Z_{i j}-Z\right)^{2}}$ 计算各个评价对象与最优 方案及最差方案的距离 $D_{i}^{+}$与 $D_{i}^{-}$。最后, 运用 公式 $B_{i}=\frac{D_{i}^{+}}{D_{i}^{+}+D_{i}^{-}}$, 计算综合评价值。其中 $\mathrm{B}$ 值越大则代表该对象的综合评价越好, 可以得 到结论如下 (见表3):

表3. 各类应灾行为的综合评价值

\begin{tabular}{ccc}
\hline 具体应灾行为 & 综合评价值 & 排序 \\
\hline 打井修水窝 & 0.9317 & 3 \\
浇灌设施准备 & 0.6480 & 5 \\
购买储水物品 & 0.4202 & 9 \\
买水 & 0.9458 & 1 \\
多样化种植 & 0.5929 & 6 \\
种植耐旱或节水作物 & 0.9347 & 2 \\
地膜覆盖 & 0.4577 & 8 \\
育苗等田间管理行为 & 0.9123 & 4 \\
购买农作物保险 & 0.3498 & 10 \\
从事多种收入活动 & 0.5203 & 7 \\
弃耕并外出务工 & 0.3066 & 11 \\
祷告祭祀神灵 & 0.0802 & 12 \\
\hline
\end{tabular}

通过以上分析可以得到, 农户认为应对早 灾最重要的行为有买水、种植耐旱或节水作物 和打井修水㝨, 同时认为购买农业保险、弃耕 外出务工和祷告祭祀神灵并不能立即有效的 应对旱灾, 减轻灾情。

\subsection{3. 结果对比分析}

通过图3可以看出, 专家和农户对 12 种不 同应灾行为重要性的评价大致相同, 但是对个
指标权重
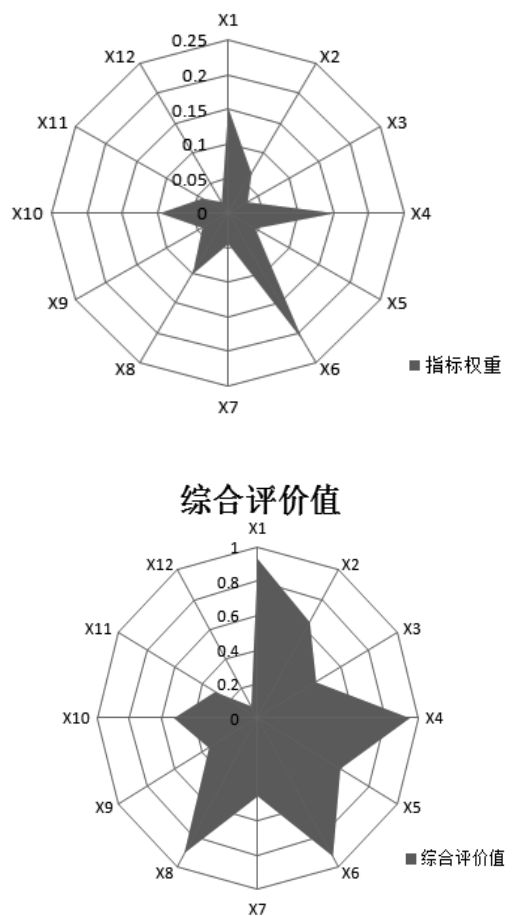

图3. 不同方法下各个应灾行为结果分析

别应灾行为如 $\mathrm{X} 3$ 购买储水物品、 $\mathrm{X} 4$ 买水、 $\mathrm{X} 5$ 多样化种植和X8育苗等田间管理行为还是存 在一定差异。表4是专家和农户对不同应灾行 为重要性评价进行了对比分析。通过对比分析 可以发现专家和农户对不同应灾行为的评价 大致一致。同时由于育苗等田间管理行为是比 较重要的应灾行为, 育苗、补栽、补种、浇灌 等田间管理行为能直接减少部分旱灾损失; 地 膜覆盖, 一方面可以增温, 另一方面可以保湿; 祷告祭祀神灵, 该行为只是一种心理寄托, 并 没有采取任何有关减灾的行为, 不能对旱灾起 到任何作用; 因此关于这 3 种应灾行为的排序 专家和农户完全一致。

但是, 对买水、从事多种收入活动和购买 储水物品等应灾行为还存在一定的差异; 买水 这一应灾行为需要考虑到附近水源情况以及 道路交通情况; 从事多种收入活动, 需要考虑 到当地经济发展水平以及文化习惯; 购买储水 物品在应灾行为中的重要程度较低, 该行为不 能直接减轻旱灾, 只有在有水的条件下, 才能 
Risk Analysis and Crisis Response in Big Data Era (RAC-16)

表4. 不同方法下应灾行为结果对比分析

\begin{tabular}{|c|c|c|c|c|c|}
\hline \multicolumn{3}{|c|}{ 研究主体: 专家 } & \multicolumn{3}{|c|}{ 研究主体: 农户 } \\
\hline \multicolumn{3}{|c|}{ 研究方法: AHP分析 } & \multicolumn{3}{|c|}{ 研究方法: TOPSIS分析 } \\
\hline 应灾行为 & 数值 & 排序 & 应灾行为 & 数值 & 排序 \\
\hline 种植耐旱或节水作物 & 0.2104 & 1 & 买水 & 0.9458 & 1 \\
\hline 打井修水窝 & 0.1511 & 2 & 种植耐旱或节水作物 & 0.9347 & 2 \\
\hline 买水 & 0.1511 & 2 & 打井修水窝 & 0.9317 & 3 \\
\hline 育苗等田间管理行为 & 0.1012 & 3 & 育苗等田间管理行为 & 0.9123 & 4 \\
\hline 从事多种收入活动 & 0.0969 & 4 & 浇灌设施准备 & 0.6480 & 5 \\
\hline 浇灌设施准备 & 0.0660 & 5 & 多样化种植 & 0.5929 & 6 \\
\hline 多样化种植 & 0.0442 & 6 & 从事多种收入活动 & 0.5203 & 7 \\
\hline 地膜覆盖 & 0.0442 & 6 & 地膜覆盖 & 0.4577 & 8 \\
\hline 购买农作物保险 & 0.0423 & 7 & 购买储水物品 & 0.4202 & 9 \\
\hline 弃耕并外出务工 & 0.0423 & 7 & 购买农作物保险 & 0.3498 & 10 \\
\hline 购买储水物品 & 0.0317 & 8 & 弃耕并外出务工 & 0.3066 & 11 \\
\hline 祷告祭祀神灵 & 0.0185 & 9 & 祷告祭祀神灵 & 0.0802 & 12 \\
\hline
\end{tabular}

发挥该行为的重要性。这3种应灾行为由于受 到自然环境状况、当地经济发展水平、农户的 生活环境和生活习惯等因素的影响, 从而使专 家和农户对这几类应灾行为的评价存在一定 程度上的差异; 同时专家和农户之间专业技能 和种植经验的差异也是影响研究结果存在差 别的原因之一。其余应灾行为的评价差异较 小。

\section{2. 应灾行为分类对比研究}

\subsection{1. 应灾行为有效性指数}

根据上文得出的各类应灾行为指标权重 值/综合评价值, 计算每个农户的应灾行为综 合权重值/综合评价值, 即应灾行为有效性指 数, 其计算公式如下所示:

$$
W=\sum_{1}^{i} a_{i} b_{i}
$$

$W$ 为应灾行为有效性指数, $i$ 为农户采取的 应灾行为种类, $a_{i}$ 为采取的应灾行为相应的权 重值/综合评价值, $b_{i}$ 为农户对不同应灾行为 重要程度的评价值。该指数的大小表示农户应 灾行为有效性的强弱。

\subsection{2. 结果对比分析}

根据组距分组方法, 首先将农户的应灾行 为分类定为 3 类一应灾行为 I 、应灾行为 II 、 应灾行为III, 即组数为 3 , 再计算组距, 组距 $=$ (最大值一最小值) / 组数。结果见表 5 所 示。

表 5 为从专家和农 户的角度出发, 通过对 应灾行为有效性指数分组来划分不同的应灾 行为。详细的分析结果如下:

（1）总体上看, 从专家的角度和从农户 的角度划分的应灾类型的数量大致相同, 特别 
Risk Analysis and Crisis Response in Big Data Era (RAC-16)

表5. 农户应灾行为有效性调查结果

\begin{tabular}{ccc|ccc}
\hline \multicolumn{2}{c}{} & \multicolumn{3}{c}{ 研究主体: 农户 } \\
\hline \multicolumn{2}{c|}{ 研究方法: AHP分析 } & & \multicolumn{3}{c}{ 研究方法: TOPSIS分析 } \\
\hline 应灾行为类型 & 应灾行为有效性指数 & 人数 & 应灾行为类型 & 应灾行为有效性指数 & 人数 \\
\hline 应灾行为 I & $2.2892 \sim 2.8073$ & $55 \%$ & 应灾行为 I & $14.8049 \sim 18.4894$ & $51 \%$ \\
\hline 应灾行为 II & $2.8074 \sim 3.3254$ & $30 \%$ & 应灾行为 II & $18.4895 \sim 22.1739$ & $31 \%$ \\
\hline 应灾行为 III & $3.3255 \sim 3.8435$ & $15 \%$ & 应灾行为III & $22.1740 \sim 25.8585$ & $18 \%$ \\
\hline
\end{tabular}

是对应灾类型 II 农户的划分接近一致; 应灾行 为 I 和应灾行为III的划分人数有一定程度的 差异。

（2）应灾行为 I 的农户测量的应灾行为 有效性指数较低, 应灾能力较弱, 该类农户采 取的应灾行为不能够及时有效的减轻旱情; 应 灾行为 II 的农户测量的应灾行为有效性指数 一般, 应灾能力属于中等水平, 该类农户采取 的应灾行为能够缓解一部分旱情; 应灾行为III 的农户测量的应灾行为有效性指数较高, 应灾 能力较强, 该类农户采取的应灾行为能够及时 有效的减轻早情。其中, 应灾行为 I 和应灾行 为 II 占据总数的绝大部分, 说明绝大多数农户 采取的应灾行为有效性属于中下水平。这一方 面可能与当地的经济发展水平有关, 另一方面 也可能与当地旱情严重, 水资源短缺有关。

（3）通过调查研究发现, 虽然属于同一种应 灾行为类型不同农户采取的应灾行为存在差 异。有的农户采取的应灾行为种类较少, 但其 采取的应灾行为重要程度比较高; 有的农户采 取的应灾行为种类较多, 但其采取的应灾行为 重要程度较低, 最终两者还是属于同一种应灾 行为类型。在问卷统计过程中发现, 不论是哪 一种风险类型的农户, 在应灾过程中都会采取 修水窑的应灾行为, 而激进型农户基本会采取 多种收入方式的应灾行为。

\section{4. 结论与讨论}

综上研究, 可得结论如下:

（1）专家和农户对 12 种不同应灾行为重 要性的评价大致相同; 但是对买水、从事多种 收入活动和购买储水物品等应灾行为还存在 一定的差异。通过调查研究, 初步认为产生这
些差异的原因主要是受到自然环境状况、当地 经济发展水平、农户的生活环境和生活习惯等 因素的影响; 同时专家和农户之间专业技能和 种植经验的差异也是影响研究结果存在差别 的原因之一。

（2）从专家的角度和从农户的角度划分 的应灾类型的数量大致相同, 特别是对应灾类 型 II 农户的划分接近一致。应灾行为 I 到应灾 行为III的应灾行为有效性指数与应灾能力依 次由弱到强。其中, 应灾行为 I 和应灾行为 II 占据总数的绝大部分, 说明绝大多数农户采取 的应灾行为有效性属于中下水平。这一方面可 能与当地的经济发展水平有关, 另一方面也可 能与当地旱情很严重, 水资源短缺有关。

（3）通过调查研究发现, 虽然属于同一 种应灾行为类型不同农户采取的应灾行为存 在差异。在分析中发现, 不论是哪一种风险类 型的农户, 在应灾过程中都会采取修水窝的应 灾行为, 而激进型农户基本会采取多种收入方 式的应灾行为。

研究不足之处主要有: (1)在问卷调查过程 中由于农户的文化程度和理解能力不高, 导致 获取数据的质量、分析和统计等工作受到影 响, 从而使得到的结果会和实际情况会有一些 出入; (2)由于研究时间、调查村落和调查样本 数量的有限性, 本文对应灾行为重要性的对比 分析和根据应灾行为有效性指数划分的应灾 行为类型其结果还需要经过长期实践不断检 验和完善。

\section{参考文献}

[1]孙雪萍,房艺,苏簤.基于旱情演变的社会应 灾过程分析一以2009-2010年云南早灾为 例.灾害学,2013,02:90-94+106. 
Risk Analysis and Crisis Response in Big Data Era (RAC-16)

[2]谢家智,周振.农业巨灾影响下农民风险态度 的行为经济学分析. 灾害学, 2009, 04: 15-19+57.

[3]刘文方,肖盛享等,隋严春,等.自然灾害链及其 断链减灾模式分析. 岩石力学与工程学 报,2006,S1:2675-2681.

[4]孙莉莉,陈爱莲,张红莉,等.台风灾害避灾行 为及影响因素的信效度分析.自然灾害学 报,2009,05:127-130.

[5]陈利,谢家智.农户对农业灾害赔偿满意度的 测量与减灾行为研究——基于 15 个省 524 户 农户的入户调查. 农业经济问题,2013, 03:56-63+111.

[6]李莉,匡昭敏,莫建飞,等.基于AHP和GIS 的 广西秋旱灾害风险等级评估. 农业工程学 报,2013,19:193-201+293.

[7] 王新民, 秦健春, 张钦礼, 等. 基于 AHP-TOPSIS评判模型的姑山驻留矿采矿方 法优选.中南大学学报(自然科学版),2013, 03:1131-1137.
[8] W.Zhu, Q.J. You. High-rise building group regional fire risk assessment model based on AHP. Journal of Risk Analysis and Crisis Response,2016,6(1):31-37.

[9] 郭金玉, 张忠涁, 孙庆云. 层次分析法的研究 与应用. 中国安全科学学报,2008,05: 148-153.

[10]刘朝亮. 层次分析法在农业系统中的应用 研究.广东农业科学,2013,13:228-232.

[11]骆正清, 杨善林. 层次分析法中几种标度的 比较.系统工程理论与实践,2004,09:51-60.

[12]孙志超, 荆绍凌, 刘文国.TOPSIS 分析法在 玉米杂交种综合评价中的应用.玉米科 学, 2006,05:49-51+55.

[13]李艳春, 黄毅斌, 王义祥, 等. 基于熵权的 TOPSIS法对福建省农业经济发展水平的 综合评价.中国农学通报,2008,09:387-390.

[14]马永仁, 徐文修, 朱美玲, 等.TOPSIS法在设 施农业种植模式综合评价中的应用. 新疆 农业科学,2010,12:2367-2370. 